\title{
Adaptive Scheduling Model in Hybrid Flowshop Production Control using Petri Net
}

\author{
$\mathrm{Hao} \mathrm{Hu}$ \\ School of Mechanical Engineering, Xinyu University, Jiangxi Xinyu 338004, \\ China \\ jwkhuhao@126.com
}

\begin{abstract}
Flowshop scheduling problems with a work shop or group shop in which the flow control shall enable an appropriate sequencing for each job and for processing on a set of machines are very significant for production control. HFS (Hybrid Flowshops) is a typical mass-customization with large product series which is widely used in the current production companies. However, as the increasing customization requirements from large number of customers, the control of HFS is really difficult. This paper introduces a Petri net-based adaptive scheduling model for easing the qualitative analysis of the production system and reducing the complexity. Resource-oriented Petri Net (ROPN) is used to establish the relation in terms of logics and behaviors of various resources, converting the dynamics control of manufacturing system into resources aspects. After the model analysis and a real-life case illustration, this model can perform good interpretation of various objective functions and real case considerations. That could be used for guiding production schedules control and automation.
\end{abstract}

Keywords: WDM, RSOA, Backscattering Interference, Crosstalk to Signal Ratio, PON

\section{Introduction}

Flowshop scheduling problems, are a class of scheduling problems with a work shop or group shop in which the flow control shall enable an appropriate sequencing for each job and for processing on a set of machines or with other resources $1,2, \ldots, m$ in compliance with given processing orders [1-2]. Especially the maintaining of a continuous flow of processing tasks is desired with a minimum of idle time and a minimum of waiting time. Hybrid Flowshops (HFS) are common manufacturing environments in which a set of $n$ jobs are to be processed in a series of $m$ stages [3]. There are a number of variants, all of which have most of the following characteristics in common: (1) the number of processing stages $m$ is at least 2; (2) each stage $k$ has $\mathbf{M}^{(\mathrm{k})}>=1$ machines in parallel and in at least one of the stages $M^{(\mathrm{k})}>1$; (3) All jobs are processed following the same and strict production flow : stage $1, \ldots$, stage $n$.

HFS is a typical mass-customization with large product series which is widely used in the current production companies [4-6]. However, as the increasing customization requirements from large number of customers, the control of HFS is really difficult. The hybrid flowshop production control (HFPC) plays critical role in the products output and profits. Thus, HFPC with high efficiency and effectiveness is very crucial, especially in facing with the large scale of production system in practical applications.

This paper takes the background of real-time production system based on the Internet of Things-enabled environment. The production resources are converted into agent-based smart objects, which have complex relations and large scale problem size. In order to tackle these challenges, this paper introduces a Petri net-based adaptive scheduling model for easing the qualitative analysis of the production system and reducing the complexity. Specifically, resource-oriented Petri Net (ROPN) is used for enable the adaptive 
scheduling model to real-time control the production activities. This model uses the Petri Net principle to establish the relation in terms of logics and behaviors of various resources, converting the dynamics control of manufacturing system into resources aspects. This innovative conversion is able to cut down the problem scales, finally to achieve adaptive control the production progress.

This paper is organized as follows. Section 2 reports on the Petri Net concepts related to manufacturing system modeling. After analyzing the HFS, section 3 illustrates the adaptive model using ROPN and rules. Section 4 uses the model to define the manufacturing Petri Net and detailed demonstrates the modeling process for production control. Section 5 discusses the qualitative analysis of the model. Section 6 concludes this paper by giving the key findings and future work.

\section{Petri Net Related to Manufacturing System Modeling}

A Petri Net is a tuple which has five elements: $P N=\left(P, T, I, O, M_{0}\right)$. Where

1. $P=\left\{p_{1}, p_{2}, \ldots, p_{m}\right\}$ is a limited set of the repository;

2. $T=\left\{t_{1}, t_{2}, \ldots, t_{n}\right\}$ is a limited set of transitions, $P \cup T \neq \varnothing, P \cap T=\varnothing$;

3. $I: P \times T \rightarrow N$ is input function, which defines the directed arc from repository to transitions, where $N=\{0,1,2, \ldots\}$;

4. $O: P \times T \rightarrow N$ is output function, which defines the directed arc from transitions to repository.

5. $M: P \rightarrow N$ presents a status vector which comes from the token quantity in all the repository in $P N$. The initial status is $M_{0}$.

The transition $t$ has a preset, which is the input repository set. ${ }^{*} t=\{p: p \in P$ and $I(p, t)>0\}$. The postset is the output repository set, $t^{*}=\{p: p \in P$ and $O(p, t)>0\}$. Similarly, the preset and postset of repository $p$ are ${ }^{*} p=\{t: t \in T$ and $O(p, t)>0\} \quad$ and $p^{*}=\{t: t \in T$ and $I(p, t)>0\}$. Given a set $S \subset P \cup T$, the preset and postset of $S$ are ${ }^{*} S=\cup_{x \in S}{ }^{*} x$ and $S^{*}=\cup_{x \in S} x^{*}$. For a $P N$, if $\left.\forall t \in T \rightarrow\right|^{*} t|=| t^{*} \mid=1$, then, $P N$ is a state machine.

In the manufacturing system, the transitions changes a lot by the driven mechanism which indicates the resources changes after passing different processing stages or machines. Thus, the firing rules could be defined as:

Definition 1. (Firing Enabled Rule). A transition $t \in T$ in $P N$, under the status $M$, for all $p \in \in^{*} t$, it meets:

$M(p) \geq I(p, t)$

Then, $t$ is enabled, which is expressed as $M[t>. t$ could be fired from a status $M$ to $M^{\prime}$ 'according to the following rules:

$$
M^{\prime}(p)=\left\{\begin{array}{c}
M(p)-I(p, t), p \in \in^{*} t \\
M(p)+O(p, t), p \in t^{*} \\
M(p), \text { otherwise }
\end{array}\right.
$$

From the definition of firing enabled rule, it is assumed that there are unlimited tokens in the repository. However, in a manufacturing system, the resources are limited. That implies each repository is able to hold certain number of tokens. Let $K(p)$ denotes the maximum quantity of tokens in a repository, the above definition could be extended that $K(p) \geq M(p)-I(p, t)+O(p, t)$ should be satisfied. 
Since the manufacturing system highly related to the time, three types of $P N$ may be involved in a HFS production. They are Timed $P N$, Transition Delayed $P N$, and Repository Delayed $P N$.

\subsection{Timed $P N$}

Timed $P N$ is a tuple which has six elements: $P N=\left(P, T, I, O, M_{0}, T I\right)$. Where $\left(P, T, I, O, M_{0}\right)$ is a $P N$;

$T I: T \rightarrow R_{0} \times\left(R_{0} \cup\{\infty\}\right)$ is the time interval function which is defined in $T \cdot R_{0}$ is not a minus time value.

For $t \in T, T I(t)=[\alpha, \beta]$, the enabled condition should meet the interval.

\subsection{Transition Delayed $P N$}

A transition delayed $P N$ has six elements: $P N=\left(P, T, I, O, M_{0}, D T\right)$, where $\left(P, T, I, O, M_{0}\right)$ is a $P N$;

$D T: T \rightarrow R_{0}$ is the time interval function which is defined in $T . R_{0}$ is not a minus time value.

For $t \in T, D T(t)=\alpha$ presents the transition $t$ may last $\alpha$ time unit. When $M[t>$, transition $t$ will happened immediately. After $\alpha$ time unit, $t$ will stop.

\subsection{Repository Delayed $P N$}

A repository delayed $P N$ has six elements: $P N=\left(P, T, I, O, M_{0}, R D\right)$, where

$\left(P, T, I, O, M_{0}\right)$ is a $P N$;

$R D: P \rightarrow R_{0}$ is the time interval function which is defined in $P . R_{0}$ is not a minus time value.

For $p \in P, D P(p)=\alpha$ presents that the token will stay in the $p$ for $\alpha$ time units.

\section{An Adaptive Scheduling Model using $P N$ in HFS}

\subsection{Problem Description}

HFS is widely used in customized production such as automotive industry, electronic products, and many mass-customization goods as well as astronautic products which are small batches or one-of-a-kind items. The characteristic of HFS is that several types of products will be processed or assembled in the shared production line or manufacturing units [7]. Take a typical HFS manufacturing for an example, figure 1 shows a typical HFS production case studied in this paper.

HFS production is usually mass-customization manufacturing mode. The due date of each customer order is fixed, thus, in the production stage, the sequence of tasks are organized by the priority. Task $\eta$ and $\theta$ have the same processing stages, however, the job instructions and operation parameters are different. Each manufacturing stage is consisted by several machines or work centers, which have the different efficiency and capacity. There are some buffers within various manufacturing stages. In this production control, the buffers are assumed unlimited. The smoothness of the plans and schedules are based on some rules, which are widely used for guiding the production. 


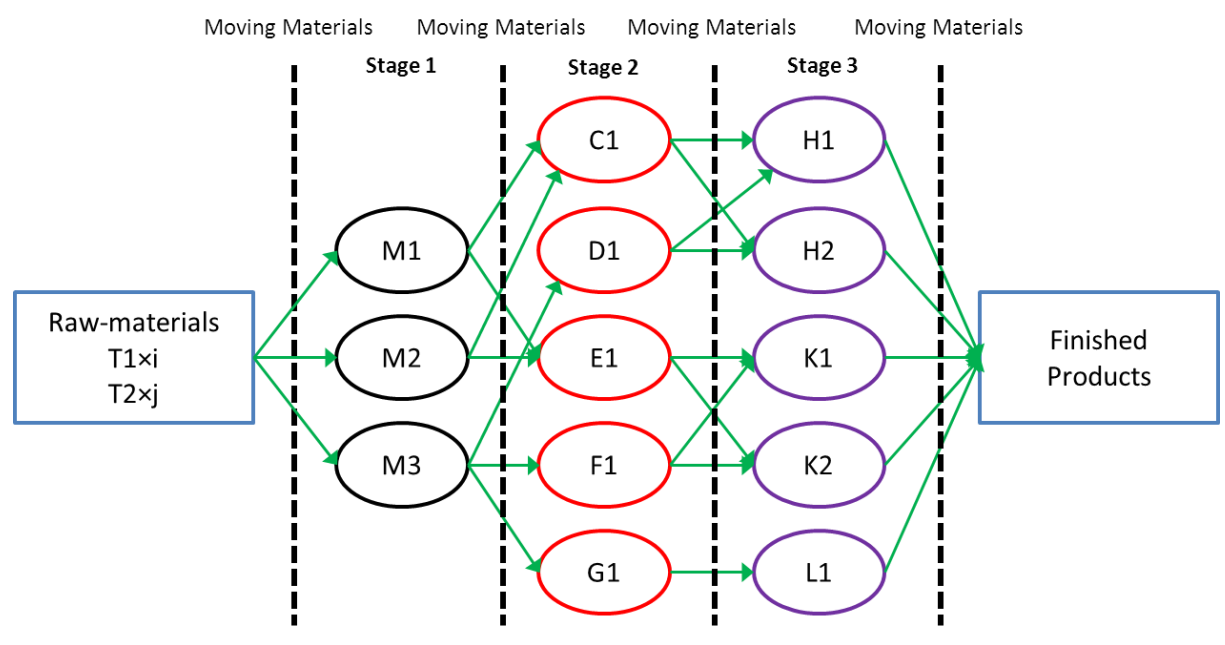

Figure 1. A Typical HFS Production Case

In this paper, some rules are specifically designed within the Petri Net. (1) A task processed in a machine with standard operation time (SOTs) is known in advanced. (2) A task must be only processed at a machine in a time. (3) A machine can only process a task at a time. (4) A task cannot be stopped once it is started.

\subsection{Manufacturing System Based on ROPN}

In the ROPN, the processing to a passive smart Agent is regarded as a resource distribution from active smart Agent, which is the resource provider [8]. Thus, the same resources are modeled as a node, which is presented by a place. This paper models the hybrid flowshop manufacturing system and the smart objects are modeled as token. The token is sent to the node so that the process will be finished. In the ROPN, the resources in each node are limited. The manufacturing system based on ROPN will use some rules to organize the hybrid flowshop production:

1. In the ROPN model, each place or node presents a type of resource. The execution of manufacturing system needs various resources like machines, tools, buffers, workers, etc. In this paper, three key resources are considered: working center (machine), logistics machine (forklift), and material buffer.

2. Each resource is equipped with Auto-ID devices so that they are converted into smart objects which are able to process the production operations. The machines and buffers are considered to be the same resources that are named as H-resource. And the logistics machines are termed as L-resource.

3. According to the same process stages each task should undertake, the processing operations must be predefined. For a specific operation, all the involved resources should be modeled.

4. For the H-resource between two operations, several conditions could be considered. Firstly, there is no buffer between two near operations. That means, the materials should enter into another machine for processing after completion. Thus the processing step should be consistent. A transit could be used for presenting the change of a job from one processing to another.

According to the rules, only the H-resources are modeled in ROPN approach so as to describe the production. A machine or working center that is an H-resource is modeled as a H-repository. Figure 2 (a) shows the modeled resource, from where, the capacity of H-repository indicates the job amount that a machine or buffer is able to handle. That means the Agent quantity in the model. There is a token 
presenting a job is in a buffer or machine. The multiple input transitions ( $t 1$ and $t 2$ ) of H-repository present that the buffer or machine will be shared by several operational processes. Multiple output transitions ( $\mathrm{t} 3$ and $\mathrm{t} 4$ ) show that different components in different jobs are delivered to different machines or buffers. For the logistics devices - G-resource, according to the 'occupy-release' repeat principle in ROPN, it is modeled as G-repository, which is shown in Figure 2 (b). The amount of tokens in G-resource presents the quantity of logistics resources. Multiple transitions connected to the G-repository reveals the sharing of logistics resource by several jobs. Thus, H-repository implies the resource and the operations provided by it. And G-repository shows the resource whose logistics operations are expressed through the connected transitions in ROPN.

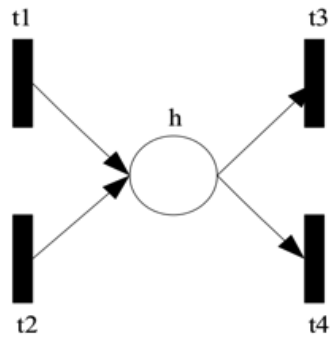

(a) H-resource

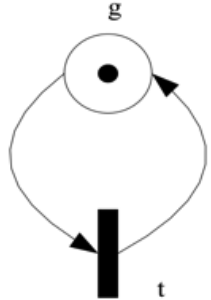

(b) G-resource

\section{Figure 2. Repository in ROPN}

\section{Adaptive Scheduling Model Based on ROPN}

According to the description of manufacturing system using Petri Net, the hybrid flowshop production system could be defined as follows:

Adaptive scheduling model is based on the manufacturing Petri Net (MPN) $M P N=\left(P, T, I, O, M_{0}, \Theta, C, D, K\right)$, where:

(1) $P=P_{H} \cup P_{G}=\left\{p_{1}, p_{2}, \ldots, p_{m}\right\}$ is a limited set of all repositories. $P_{H}$ presents the H-repository and $P_{G}$ is a set of G-repository. $P_{H} \cap P_{G}=\varnothing$. $\left.\forall t \in T \rightarrow\right|^{*} t \cap P_{H}|=| t^{*} \cap P_{H} \mid=1$ and $\left.\right|^{*} t \cap P_{G}|=| t^{*} \cap P_{G} \mid \leq 1$.

(2) $T=\left\{t_{1}, t_{2}, \ldots, t_{n}\right\}$ is a set of transitions. $P \cup T \neq \varnothing$ and $P \cap T=\varnothing$.

(3) $I(p, t): P \times T \rightarrow N$ is an input function which defines the directed arc from a repository to a transition. $N=\{0,1,2, \ldots\}$.

(4) $O(p, t): P \times T \rightarrow N$ is an output function which defines the directed arc from a transition to a repository.

(5) $\Theta: \Theta(\theta)=($ COLOR, KNOW $)$ is the knowledge function which is carried by token $\theta . C O L O R \in\left\{c_{1}, c_{2}, \ldots, c_{u_{i}}\right\}$ is the information of colors. KNOW presents the knowledge vector carried by smart token.

(6) $M: P \rightarrow N$ is a status vector from the token quantity in all the repository with MPN. $M\left(P_{i}\right)$ means the total amount of tokens in repository $p_{i} \cdot M\left(p_{i}, c_{j}\right)$ reveals the quantity of tokens with $c_{j}$ color in repository $p_{i} \cdot M_{0}$ presents the initial status of MPN. 
(7) $C(p): P \rightarrow N^{+}$is the capacity function of MPN. It meets $\forall p_{i} \in P \rightarrow M\left(p_{i}\right) \leq C\left(p_{i}\right)$ where $N^{+}=\{1,2, \ldots\}$. That means the token amount $M\left(p_{i}\right)$ is less than the capacity $C\left(p_{i}\right)$ of $p_{i}$.

(8) $D(p, \theta): P \times \Theta \rightarrow R_{0}$ is a time function defined in repository $p \cdot R_{0}$ is a time value which is above 0 and means the stay time of token $\theta$ in $p . \forall p_{i} \in P_{H}$, $D\left(p_{i}, \theta_{J}\right)=R_{0}$ is the stay time of $\theta_{J}$ in $p_{i} \cdot \forall p_{i} \in P_{G}, D=0$.

(9) $K(p, \theta, \tau)=\{t \mid t \in T\}$ is the knowledge function.

(10) $P T(M, t, \theta, \tau): P T(M, t, \theta, \tau) \rightarrow[0,1]$ presents the possibility function at time $\tau$ under $M[t>$ condition transition $t$ driven by $\theta$ for firing.

From the model, it can be seen that, in the MPN, repository $P$ is divided into two types and the colors are used for delivering the information according to the changes of the transitions. Thus, the resources adjustment could be carried out adaptive to schedule the efficiency of jobs. For $p_{i} \in P_{H}, C\left(p_{i}\right)$ is the color set presenting the job types. $\forall p_{i} \in P_{G} \rightarrow\left|C\left(p_{i}\right)\right|=1$ means the logistics resources are not differentiated by colors. From $\left.\forall t \in T \rightarrow\right|^{*} t \cap P_{H}|=| t^{*} \cap P_{H} \mid=1$, it could be observed that $\left(P_{H}, T, I, O, M_{0}\right)$ is a status machine, from which the possibility of the occurrence of transitions could be controlled. Thus, the schedules could be based on the colors and transitions which are real-time supervised.

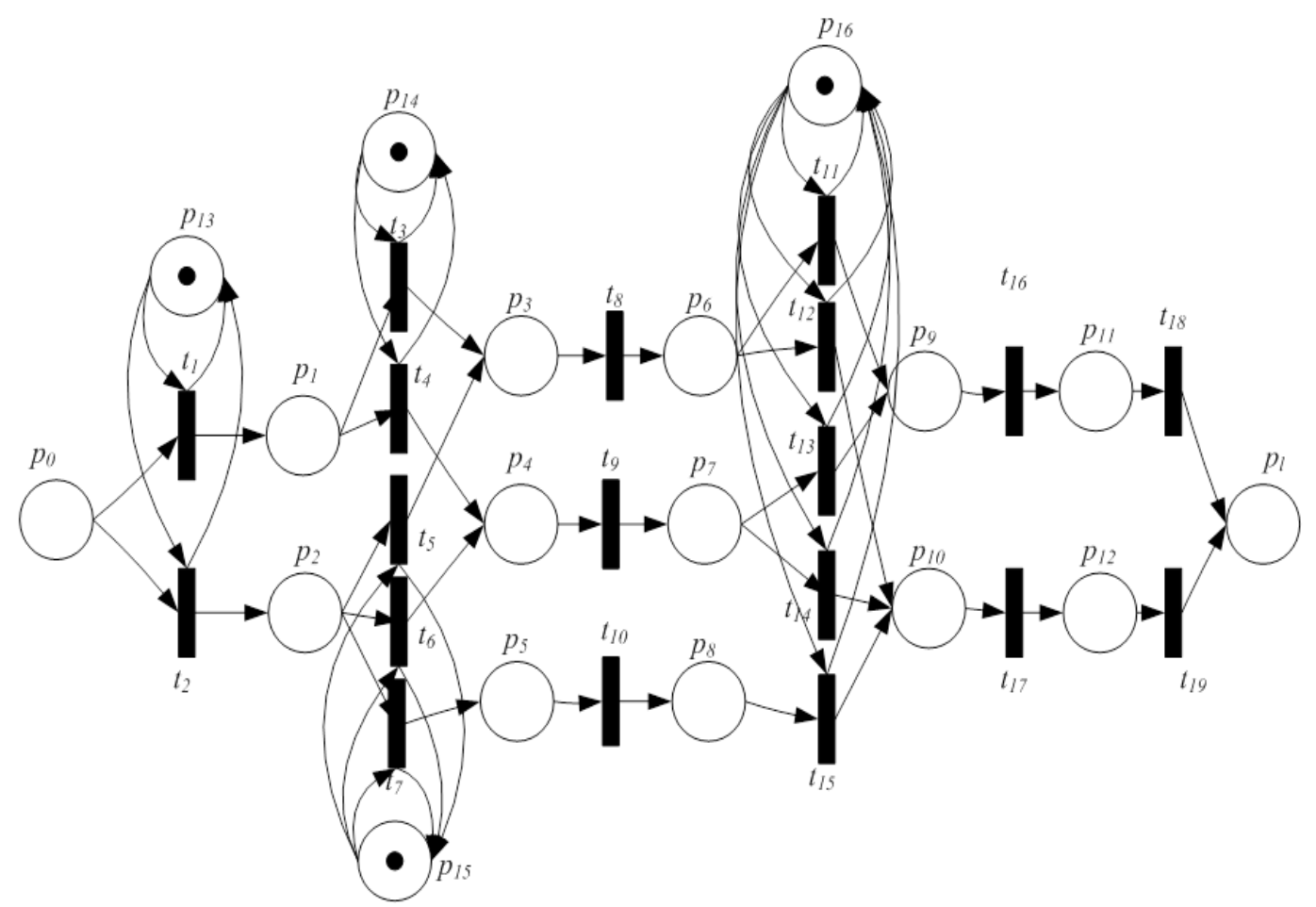

Figure 3. ROPN Model for Hybrid Flowshop Production

Based on the above model, a Petri Net is established for descripting the previous mentioned typical hybrid flowshop production case. Figure 3 shows the ROPN model for hybrid flowshop production. The adaptive scheduling is modeled as follows: 
(1) Confirm the manufacturing machines and buffers according to the process and operations. Let $S T E P S=\left\{\Delta_{1}, \Delta_{2}, \ldots, \Delta_{n}\right\}$ presents the set of operations. $\Delta_{1} \rightarrow \Delta_{2} \rightarrow \ldots \rightarrow \Delta_{n}$ is the operational path.

(2) Model the H-repository according to the STEPS

a. Define the $\mathrm{H}$ set in $\Delta_{i} U^{i}=\left\{U_{1}^{i}, U_{2}^{i}, \ldots, U_{m}^{i}\right\}$;

b. Establish a repository $p_{0}$ presenting the initial position, $k=1$;

c. For $i=1$ to $n$, repeat

i. Select $\Delta_{i}$, let $j=0, \Gamma_{i}=\varnothing$;

ii. $\quad j=j+1$, select a H-repository $U_{j}^{i}$ from $\Delta_{i}$;

iii. Let $k=k+1, \Gamma_{i}=\Gamma_{i} \cup\left\{U_{j}^{i}\right\}$, establish a H-repository as $p_{k}$. $\forall p_{x} \in \Gamma_{i-1}$, if there is a deliver relationship between $p_{x}$ and $p_{k}$, then add a transition $t$. A directed arc is add to $\left(p_{x}, t\right)$ and $\left(t, p_{k}\right)$ as well as the input and output functions $I\left(p_{x}, t\right)$ and $O\left(p_{k}, t\right)$.

iv. If $\Gamma_{i}<U^{i}$ go to ii.

d. Establish a repository $p_{l}$, for $\forall p_{x} \in U^{n}$, add a transition $t$ between $p_{x}$ and $p_{l}$. Add directed $\operatorname{arc}\left(p_{x}, t\right)$ and $\left(t, p_{l}\right)$, define $I\left(p_{x}, t\right)$ and $O\left(p_{l}, t\right)$.

(3) Model the logistics capacity G-repository:

a. For each logistics resource $r_{i} \in R$;

i. Let $k=k+1$, establish a $\mathrm{G}$ repository, $p_{k}$;

ii. According to the requirement of $r_{i}$ for $\mathrm{G}$, connect $p_{k}$ and corresponding $t_{x}$, which is a transition between $p_{y}$ and $p_{z}$. Add $\left(p_{k}, t_{x}\right)$ and $\left(t_{x}, p_{k}\right)$ between $p_{k}$ and $t_{x}$.

b. Define the initial parameters in MPN:

i. Input the color token at $p_{0}, M_{0}\left(p_{0}\right)=\left\{\theta_{1}, \theta_{2}, \ldots \theta_{u^{0}}\right\}$ presents the initial token amount in MPN;

ii. For $\forall p_{x} \in P_{G}$, put the token presenting the logistics resource in $p_{x}$

iii. Initial the possibility function $P T$;

\section{Model Analysis and Discussions}

The scheduling model for production control based on the ROPN mentioned in the previous section could be interpreted with real-life cases. $p_{0}$ and $p_{1}$ could be merged as $p_{0}$ so that a strong connected Petri net could be built up. In the manufacturing system, the resources are used in single direction mode. If there is no isolated node in the system, the adaptiveness of this model could be ensured. Meanwhile, as the limited resources in the system, MPN has the upper boundary.

This paper analyzes the objective functions from two aspects. Firstly, minimization of the makespan is considered. It aims to finish all the jobs under minimal time interval [9]. 
In the ROPN model, it means that all the tokens should arrived at $p_{l}$ through MPN system with the shortest time expenditure. Assume that the time starts at 0 , then the objective function could be expressed as:

$\min ($ Makespan $)=\min \left(C_{\max }\right)=\min \left(F\left(M_{0} \rightarrow M_{l}\right)\right)$

Where $F\left(M_{0} \rightarrow M_{l}\right)$ is the total time cost from the starting status $M_{0}$ to finished status $M_{l}$.

Secondly, minimization of WIP (work-in-progress) is concerned. In a large hybrid flowshop production system, the WIP inventory is high, resulting the high occupation of cash flow [10]. Thus, the objective is to cut down the WIP inventory and finally release the cash. The objective could be expressed as:

$$
\min (W I P)=\min \left(\sum_{p_{i} \in P_{H}} \int_{0}^{T} M\left(p_{i}\right) d t\right)
$$

The WIP items are only stored in the H-resource. $p_{i} \in P_{H}$ reveals to calculate the token amount in the H-repository.

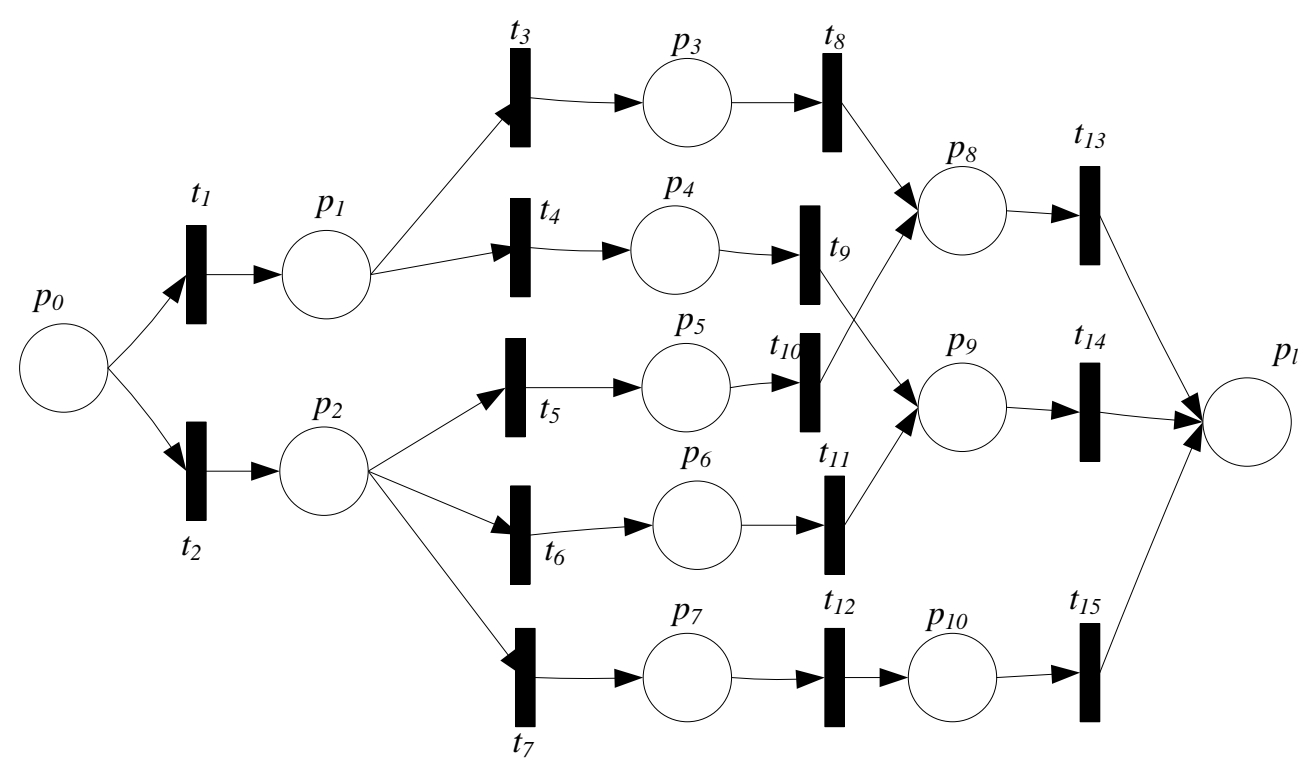

Figure 4. ROPN Model for Bronze Plate Production

Take the bronze plate for example to illustrate the adaptive model. Figure 4 shows the ROPN model which has the following parameters:

(1) $P=P_{H} \cup P_{G}, P_{H}=\left\{p_{1}, p_{2}, p_{3}, p_{4}, p_{5}, p_{6}, p_{7}, p_{8}, p_{9}, p_{10}\right\}, P_{G}=\varnothing$;

(2) Assume that this manufacturing system produces five categories of bronze plate. $\forall \theta \in \Theta, \operatorname{COLOR}=\left\{c_{1}, c_{2}, c_{3}, c_{4}, c_{5}\right\}$ is the color attribute set of the smart token. The color of the tokens will not change within the transitions.

(3) $p_{i} \in P, t_{j} \in T$, then $\left(\exists\left(p_{i}, t_{j}\right) \in I \vee \exists\left(p_{i}, t_{j}\right) \in O\right) \rightarrow I\left(p_{i}, t_{j}\right)=1$ and $O\left(p_{i}, t_{j}\right)=1$. That means in the weight definition of $I$ and $O$, if there is a directed arc between $p_{i}$ and $t_{j}$. Then, all the quantity of tokens is 1 .

(4) $\left\{K\left(p_{i}\right)\right\}^{T}=\{2,1,10,10,5,5,5,2,2,1\} \quad(i \in[1,10]), p_{3}, p_{4}, p_{5}, p_{6}, p_{7}$ is $\mathrm{H}-$ repository with the maximum capacity.

In a real-life bronze plate production system, different categories have different length, width, and other items. Jobs are release as "book". Each job has different "book" quantity. 
Once the job is started, it cannot be stopped until it is completely finished. The stay time of smart token mapped from the jobs is the unit "book" processing time multiplies the quantity. Unit "book" processing time is calculated by $D\left(p_{1}\right)=\left\{D\left(C\left(P_{1}\right)\right)\right\}^{T}=\{2,3,3,4,2\}^{T} /$ book $D\left(p_{2}\right)=\left\{D\left(C\left(P_{2}\right)\right)\right\}^{T}=\{3,2,2,3,2\}^{T} /$ book. In an operation, the efficiency of M and $\mathrm{N}$ machines are different:

$$
D\left(p_{3}\right)=\left\{D\left(C\left(P_{3}\right)\right)\right\}^{T}=D\left(p_{6}\right)=\left\{D\left(C\left(P_{6}\right)\right)\right\}^{T}=D\left(p_{7}\right)=\left\{D\left(C\left(P_{7}\right)\right)\right\}^{T}=\{5,5,5,5,5\}^{T}
$$

The transfer and buffer time within different flowshops is accumulated in buffer time. Each token in the buffer cost the same time unit 5 with no relationship of quantity.

$$
D\left(p_{4}\right)=\left\{D\left(C\left(P_{4}\right)\right)\right\}^{T}=D\left(p_{5}\right)=\left\{D\left(C\left(P_{5}\right)\right)\right\}^{T}=\{12,12,12,12,12\}^{T} \text { is the transfer }
$$

time across different flowshops. Tokens in the buffer have the same transfer time 12.

$$
\begin{aligned}
& D\left(p_{8}\right)=\left\{D\left(C\left(P_{8}\right)\right)\right\}^{T}=\{3,4,4,3,5\}^{T} / \text { book } \\
& D\left(p_{9}\right)=\left\{D\left(C\left(P_{9}\right)\right)\right\}^{T}=\{4,5,4,3,5\}^{T} / \text { book } \\
& D\left(p_{10}\right)=\left\{D\left(C\left(P_{10}\right)\right)\right\}^{T}=\{3,4,5,4,3\}^{T} / \text { book }
\end{aligned}
$$

\section{Conclusion}

This paper introduces an adaptive scheduling model in hybrid flowshop production control based on the Petri Net. This model is based some proposed definitions in the background of hybrid flowshop production which is widely used in the manufacturing fields. Using the definitions in Petri Net, a real-life case is considered for modeling given the key concept of ROPN, which is used for establishing the manufacturing Petri net. From a specific bronze plate manufacturing case, this model is examined for demonstrate the adaptive schedule using the tokens passes and time cost in the MPN.

Future research directions will be carried out to enhance the model. First of all, this paper considers two types of resources: H and G. However, more types of resources like electricity and water will be involved mainly in production. How to model this main cost in the Petri Net will be further concerned. Secondly, this model could be implemented into a decision support system (DSS) which is able to assist the decision-makers to work out precise schedules.

\section{References}

[1] I. Budinská, T. Kasanický and J. Zelenka, "Production planning and scheduling by means of artificial immune systems and particle swarm optimisation algorithms", International Journal of Bio-Inspired Computation, vol. 4, (2012), pp. 237-248.

[2] R. Y. Zhong, Q. Y. Dai, T. Qu, G. J. Hu and G. Q. Huang, "RFID-enabled Real-time Manufacturing Execution System for Mass-customization Production", Robotics and Computer-Integrated Manufacturing, vol. 29, (2013), pp. 283-292.

[3] A. Rajabinasab and S. Mansour, "Dynamic flexible job shop scheduling with alternative process plans: an agent-based approach", The International Journal of Advanced Manufacturing Technology, vol. 54, (2011), pp. 1091-1107.

[4] Q. Y. Dai, R. Y. Zhong, G. Q. Huang, T. Qu, T. Zhang and T. Y. Luo, "Radio frequency identificationenabled real-time manufacturing execution system: a case study in an automotive part manufacturer", International Journal of Computer Integrated Manufacturing vol. 25, (2012), pp. 51-65.

[5] R. Y. Zhong, Z. Li, A. L. Y. Pang, Y. Pan, T. Qu and G. Q. Huang, "RFID-enabled Real-time Advanced Planning and Scheduling Shell for Production Decision-making", International Journal of Computer Integrated Manufacturing., vol. 26, pp. 649-662, (2013).

[6] R. Bhatnagar, P. Mehta and C. Chong Teo, "Coordination of planning and scheduling decisions in global supply chains with dual supply modes", International Journal of Production Economics, vol. 131, (2011), pp. 473-482.

[7] V. Bharathi, D. Pramod and R. Raman, "A fuzzy petri-net based conceptual model for risk prediction in enterprise resource planning acquisition decisions for small and medium enterprises", Journal of Computer Science, vol. 9, (2013). 
[8] N.Q. Wu, F. Chu, C. Chu and M. Zhou, "Petri net modeling and cycle-time analysis of dual-arm cluster tools with wafer revisiting", Systems, Man, and Cybernetics: Systems, IEEE Transactions on, vol. 43, (2013), pp. 196-207.

[9] F. S. de Boer, M. Bravetti, I. Grabe, M. Lee, M. Steffen and G. Zavattaro, "A petri net based analysis of deadlocks for active objects and futures", in Formal Aspects of Component Software, ed: Springer, (2013), pp. 110-127.

[10] R. Y. Zhong, Q. Y. Dai, K. Zhou and X. B. Dai, "Design and Implementation of DMES Based on RFID", in 2nd International Conference on Anti-counterfeiting, Security and Identification, Guiyang, vol. 475-477, (2008), pp. 475-477.

\section{Author}

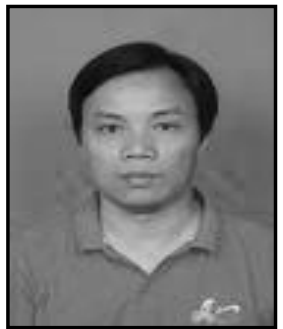

Hu Hao is a lecturer in School of Mechanical Engineering, Xinyu College. His bachelor was obtained from Nanchang University in Electronic and Communication Engineering 2008 respectively. His research interests include communication system, control systems, and computer networks. He has been published over 10 articles in international journals and conferences. 